\title{
Synchronous presentation of primary renal adenocarcinoma and contralateral ureteric metastasis
}

\author{
I Pearce, J M V Harney, R F T McMahon
}

\begin{abstract}
The synchronous presentation of a primary renal adenocarcinoma and contralateral uretic metastasis is discussed. The pattern of immunohistochemical staining confirmed that the contralateral ureteric obstruction was the result of a metastasis from the primary renal adenocarcinoma. (F Clin Pathol 2000;53:639-640)
\end{abstract}

Keywords: primary renal adenocarcinoma; contralateral ureteric metastasis

\section{Case report}

A 67 year old man presented to the physicians with general malaise and confusion. Biochemical analysis confirmed chronic renal failure with a serum creatinine of $1345 \mu \mathrm{mol} /$ litre (normal range, 50-120) and urea of $52.6 \mathrm{mmol} /$ litre (normal range, 3.5-8) and a metabolic alkalosis as a result of vomiting. An ultrasound scan of his abdomen revealed gross bilateral hydronephrosis, and nephrostomies were inserted. The right nephrostomy drained pus and after decompression ceased to drain, whereas the left drained clear urine and continued to do so.
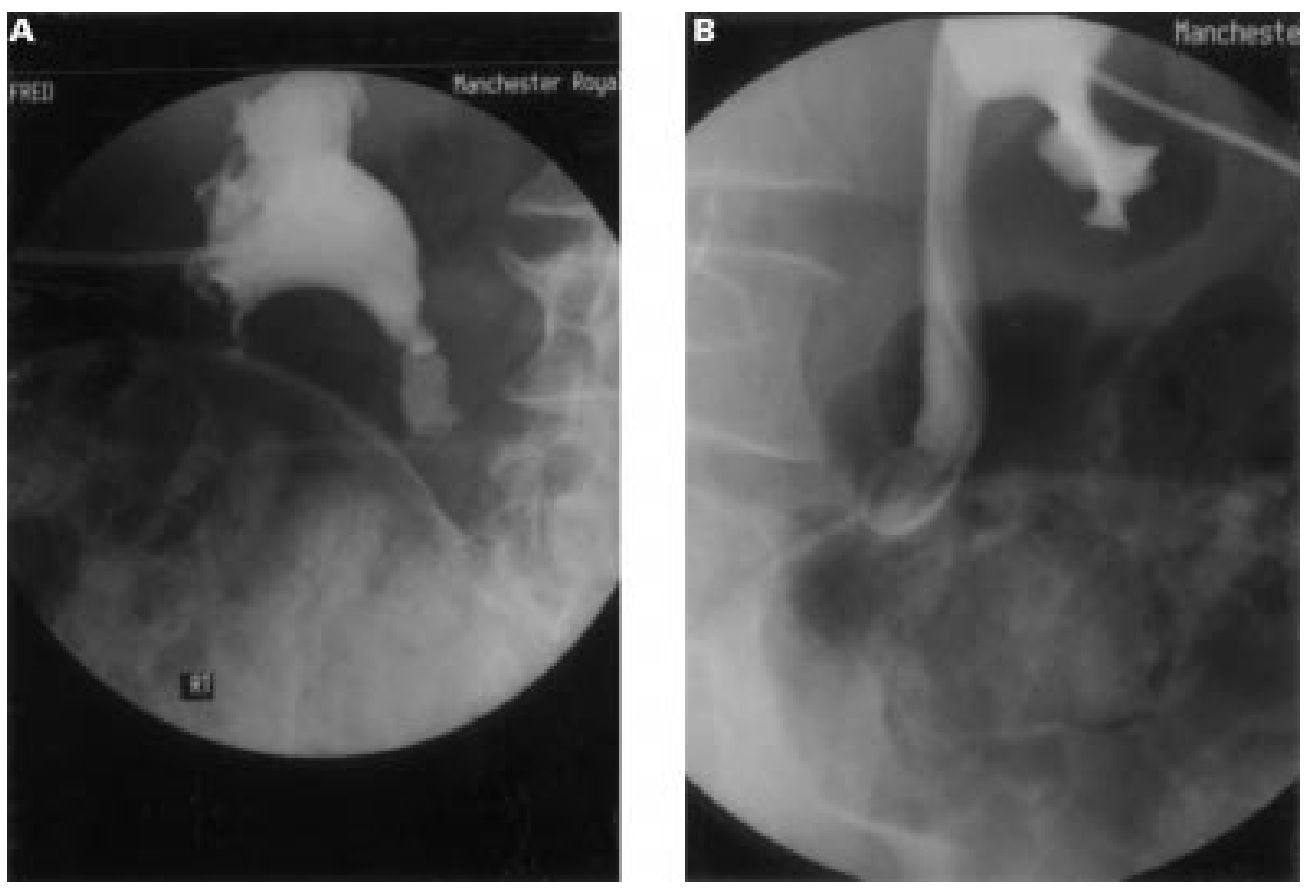

Figure 1 Bilateral nephrostograms showing obstruction of the right pelvicalyceal system at the level of L3 and almost complete non-visualisation of the left side.

Nephrostograms demonstrated obstruction of the right ureter at the level of L4 and obstruction of the left ureter in the distal third with no contrast entering the bladder (fig 1).

Computed tomography (CT) scanning with contrast revealed a mass in the lower pole of the right kidney but failed to show any cause for the left ureteric obstruction. Biopsy of the mass confirmed a primary renal cell carcinoma showing a predominantly clear cell pattern, with moderate nuclear pleomorphism and occasional granular cells (fig 2). The tumour cells contained glycogen (periodic acid Schiff (PAS) positivity cleared with diastase) and were immunohistochemically reactive with a range of cytokeratins (CAM5.2, AE1/AE3), whereas vimentin staining was negative within the tumour.

Cystoscopy and left ureteroscopy were performed and two superficial transitional cell carcinomas (G1 pTa) of the bladder were found. The ureteroscope would not pass the obstruction at the distal third of the left ureter.

Unfortunately, because of chronic malnutrition and self neglect, the patient was not fit for definitive surgery bilaterally with prolonged operative time. With this in mind, the left ure-

Accepted for publication 6 August 1999
Manchester Royal Infirmary, Oxford M13 9WL, UK I Pearce

J M V Harney R F T McMahon

Correspondence to: Mr Harney

email: urology@medit. cmht.nwest.nhs.uk 


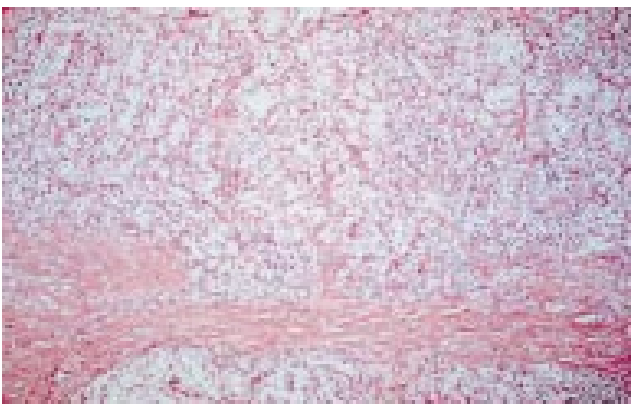

Figure 2 Renal cell carcinoma of predominantly clear cell type (haematoxylin and eosin; magnification, $\times 120$ ).

ter was explored. At surgery, pronounced thickening of the distal left ureter was noted. This was resected and the ureter re-implanted using a Psoas hitch.

Histology of the resected ureter revealed infiltration of the wall by a malignant tumour (fig 3). The tumour was composed of a mixture of clear and granular cells (fig 4) with identical histochemical (PAS positivity cleared with diastase) and immunohistochemical (CAM5.3 positive, AE1/AE3 positive, and vimentin negative) patterns to the primary renal carcinoma.

\section{Comment}

There are many cases of ureteric metastases in the literature but only 48 from renal primaries, 40 involving the ipsilateral ureter and eight the contralateral ureter. Of these eight, six were metachronous. ${ }^{1}$ Therefore, our case is only the third case to be reported of a contralateral ureteric metastasis from a renal primary adenocar-

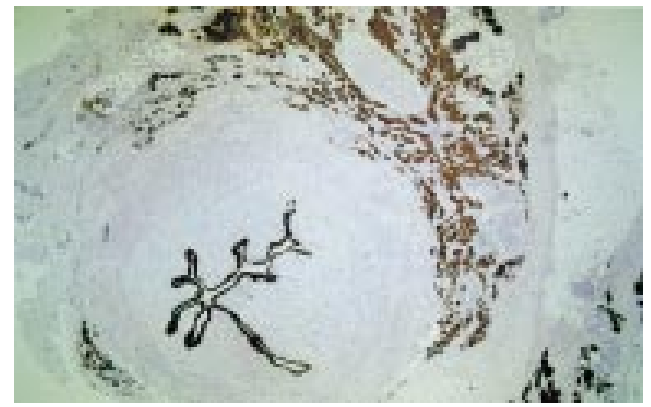

Figure 3 Lower end of ureter demonstrating infiltration of wall by a malignant tumour shown on immunostaining with CAM5.2 (magnification, $\times 11$ ).

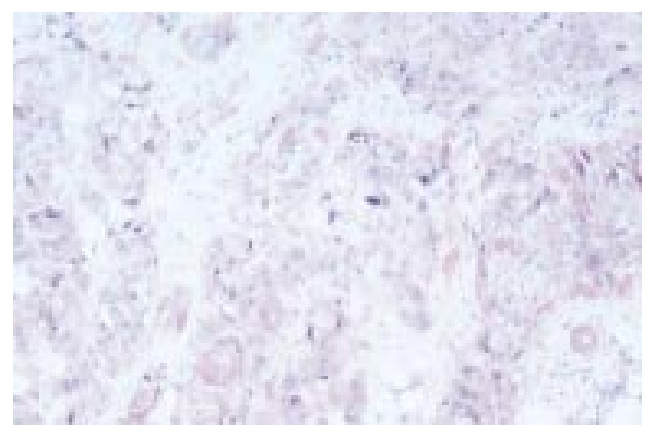

Figure 4 Wall of ureter infiltrated by a carcinoma showing both clear and granular cell patterns (haematoxylin and eosin; magnification, $\times 120$ )

cinoma presenting synchronously, although in contrast to the previous cases this one did not present with haematuria.

1 Esrig D, Kanellos AW, Freeman JA, et al. Metastatic renal Esrig D, Kanellos AW, Freeman JA, et al. Metastatic renal
cell carcinoma to the contralateral ureter. Urology cell carcinoma 